Discrete Comput Geom 34:97-109 (2005)

DOI: $10.1007 / \mathrm{s} 00454-004-1129-\mathrm{z}$

Geometry

\title{
Improved Dense Packings of Congruent Squares in a Square
}

\author{
Thierry Gensane and Philippe Ryckelynck \\ LMPA, Université du Littoral, \\ 50 rue F. Buisson, BP699, 62228 Calais Cedex, France \\ \{gensane,ryckelyn\}@1mpa.univ-littoral.fr
}

\begin{abstract}
Let $s_{n}$ be the side of the smallest square into which it is possible to pack $n$ congruent squares. In this paper we link $s_{n}$ to the supremum of the maximal inflation $\omega(\mathcal{C})$ of admissible configurations $\mathcal{C}$. The computation and the properties of $\omega(\mathcal{C})$ and related functions give rise to an algorithm similar to the billiard approach used to pack congruent disks or spheres in a bounded domain. We improve the best known packings of $n$ equal squares for $n=11,29$ and 37, and give an alternative optimal packing of 18 squares.
\end{abstract}

\section{Introduction}

Erdôs and Graham [2] initiated the problem of maximizing the area sum of packings of an arbitrary square by unit squares. The present paper deals with the research of the densest packing of a given number $n$ of unit squares in a larger square. As usual, we denote by $s_{n}$ the side of the smallest square into which one can pack $n$ unit squares.

Kearney and Shiu [7] proved that $s_{6}=s_{7}=s_{8}=s_{9}=3$. Stromsquist [10] showed that $s_{10}=3+1 / \sqrt{2}$ and $s_{11} \geq 2+4 / \sqrt{5}$. Friedman [3] gave an interesting survey, with emphasis on the technique of "unavoidable" points which allows him to give short proofs of optimality for $n=2,3,5,8,15,24$ and 35; he also recorded in two tables the upper and lower bounds for $s_{n}$.

In the survey [3], Friedman does not give any light on the underlying methods or algorithms which have been used to produce optimal configurations. We give here a heuristic approach similar to the billiard algorithms which have been used in the case of disk packings by Graham and Lubachevsky [8], [5], see also [1] and [6]. The billiard approach for sphere packings in a cube is treated in [4].

The billiard algorithm is implicitly based on the fact that, if $K$ is a square, the disk packing problem is equivalent to the maximal separation problem [9]: how to find an 
$n$-tuple $\mathcal{P}=\left(p_{1}, \ldots, p_{n}\right) \in K^{n}$ which attains

$$
\delta_{n}=\max _{\mathcal{P}} f(\mathcal{P}) \quad \text { where } \quad f(\mathcal{P})=\min _{1 \leq i<j \leq n}\left|p_{i-} p_{j}\right| .
$$

In fact, the quantity $f(\mathcal{P})$ represents the maximal diameter of $n$ non-overlapping disks centered at the points $p_{i}$. Similarly, we define in Proposition 3 a function $\omega(\mathcal{C})$ which gives the maximal half-diagonal of a configuration $\mathcal{C}$ of $n$ non-overlapping squares contained in the square $[-L, L]^{2}$. The choice of the half-diagonal as a measure of the size of $\mathcal{C}$ is clear from Remark 4. The adaptation of the billiard algorithm for sphere packings [4] into an algorithm for squares packing follows mainly from substitution of the function $\omega$ for the function $f$.

In Section 2 we fix notation, quote simple facts from euclidean geometry and introduce the manifold of admissible configurations $\mathbf{Q}_{n, c}$. In Sections 3-5 we compute some necessary functions $\varphi, \psi: \mathbf{Q}_{n, c} \rightarrow \mathbb{R}_{+}$to define the function $\omega$ and to link $s_{n}$ with $\omega$. In Section 5 we also introduce the geometric graph associated with any admissible configuration. In Section 6, we give some hints on the code that we wrote to compute dense packings. Our results are detailed in Section 7 for the cases $n=11,17,18,29$ and 37 .

\section{Notation}

Throughout the paper, $n \geq 2$ is a fixed integer and $L>0$ is fixed. We denote by $B(\mathbf{x}, \varepsilon)=$ $\left\{\mathbf{y} \in \mathbb{R}^{2}: d(\mathbf{x}, \mathbf{y})<\varepsilon\right\}$ the open ball of radius $\varepsilon$ centered at $\mathbf{x}$. The group of displacements of the plane $\mathfrak{D} \approx S O(2, \mathbb{R}) \bowtie \mathbb{R}^{2}$ is diffeomorphic to $\left\{(\alpha, \beta, \eta) \in \mathbb{R}^{2} \times \mathbb{R} / 2 \pi \mathbb{Z}\right\}$. The displacement $g: \mathbb{R}^{2} \rightarrow \mathbb{R}^{2}$ with parameters $(\alpha, \beta, \eta)$ acts as follows on $\mathbb{R}^{2}$ :

$$
g \cdot(x, y)=(\alpha, \beta, \eta) \cdot(x, y)=(\alpha+x \cos \eta-y \sin \eta, \beta+x \sin \eta+y \cos \eta) .
$$

The law in the group $(\mathfrak{D}, \circ)$ is then

$$
\begin{aligned}
\left(\alpha^{\prime}, \beta^{\prime}, \eta^{\prime}\right) \circ\left(\alpha^{\prime \prime}, \beta^{\prime \prime}, \eta^{\prime \prime}\right) & =\left(\alpha^{\prime \prime \prime}, \beta^{\prime \prime \prime}, \eta^{\prime}+\eta^{\prime \prime}\right) \quad \text { with } \\
\left(\alpha^{\prime \prime \prime}, \beta^{\prime \prime \prime}\right) & =\left(\alpha^{\prime}, \beta^{\prime}, \eta^{\prime}\right) \cdot\left(\alpha^{\prime \prime}, \beta^{\prime \prime}\right) .
\end{aligned}
$$

Given $(a, b) \in \mathbb{R}^{2}$ and $\theta \in[0, \pi / 2]$, we use the notation $q_{a, b, \theta, c}$ for the square centered at $(a, b)$ at angle $\theta$, which has side $c \sqrt{2}$ and diagonal $2 c$. This square has the four vertices $(a, b)+c\left(\cos \theta_{i}, \sin \theta_{i}\right)$, with $\theta_{i}=\theta+i(\pi / 2)$ for $i$ from 1 to 4 ; its boundary is denoted as usual by $\partial\left(q_{a, b, \theta, c}\right)$. For any number $c>0$, we denote by $\mathbf{Q}_{c}$ the set of squares of side $c \sqrt{2}$ in $\mathbb{R}^{2}$, with the topology induced by $\mathbb{R}^{2} \times S^{1}$. The space $\mathbf{Q}_{c}$ is locally compact and simply connected and contractible.

The square $q_{0,0,0, c}$ is defined by the inequality $|x|+|y| \leq c$. We find the inequality of a square $q_{a, b, \theta, c} \in \mathbf{Q}_{c}$ by determining $g_{\alpha, \beta, \eta} \in \mathfrak{D}$ which maps the square $q_{a, b, \theta, c}$ onto $q_{0,0,0, c}$. We get $(\alpha, \beta, \eta)=(-a \cos \theta-b \sin \theta, a \sin \theta-b \cos \theta,-\theta)$. Then $(x, y) \in$ $q_{a, b, \theta, c}$ if and only if

$$
|(x-a) \cos \theta+(y-b) \sin \theta|+|-(x-a) \sin \theta+(y-b) \cos \theta| \leq c .
$$


We define $h_{k}\left(q_{a, b, \theta, c}\right)=q_{a, b, \theta, k c}$ to be the homothetic of the square $q_{a, b, \theta, c}$ with respect to its center.

Given $g \in \mathfrak{D}$, the direct image set $g\left(q_{a, b, \theta, c}\right)=q_{a^{\prime}, b^{\prime}, \theta^{\prime}, c}$ is a square centered at $g(a, b)$; we use the notation $g \cdot q=g(q)$. We note that $\mathfrak{D}$ acts simply and transitively on $\mathbf{Q}_{c}$ and $\mathbf{Q}_{c} / \mathfrak{D}=\left\{q_{0,0,0, c}\right\}$. The boundaries of the squares obtained by those operations are related by $\partial\left(g \cdot q_{a, b, \theta, c}\right)=g\left(\partial\left(q_{a, b, \theta, c}\right)\right)$ and we have $\partial\left(h_{k}\left(q_{a, b, \theta, c}\right)\right)=h_{k}\left(\partial\left(q_{a, b, \theta, c}\right)\right)$.

We define the manifold of ordered configurations of $n$ non-overlapping squares of diagonal $2 c$ contained in $[-L, L]^{2}$ as the set

$$
\begin{aligned}
\mathbf{Q}_{n, c}= & \left\{\mathcal{C}=\left(q_{1}, \ldots, q_{n}\right) \in \mathbf{Q}_{c}^{n}, q_{i} \subset[-L, L]^{2} \text { for all } i\right. \text { and } \\
& \left.\operatorname{int}\left(q_{i}\right) \cap \operatorname{int}\left(q_{j}\right)=\emptyset \text { for } i<j\right\} .
\end{aligned}
$$

When $\mathcal{C} \in \mathbf{Q}_{n, c}$, we say that $\mathcal{C}$ is an admissible configuration.

Finding the real number $s_{n}$ is equivalent to the maximization of $c>0$ such that $\mathbf{Q}_{n, c}$ is not empty. For $L=1$, we note that $\max \left\{c: \mathbf{Q}_{n, c} \neq \emptyset\right\}=\sqrt{2} / s_{n}$.

We define the homothetic of an admissible configuration $\mathcal{C}=\left(q_{1}, \ldots, q_{n}\right)$ by $h_{k}(\mathcal{C})=$ $\left(h_{k}\left(q_{1}\right), \ldots, h_{k}\left(q_{n}\right)\right)$, and we note that it makes sense for $0<k \leq \omega(\mathcal{C}) / c$ where $\omega(\mathcal{C}) / c \geq 1$.

We define an embedding of $\mathbf{Q}_{n, c}$ into $\left([-L, L]^{2} \times(\mathbb{R} /(\pi / 2) \mathbb{R})\right)^{n}$ as follows: the requirements $\operatorname{int}\left(q_{i}\right) \cap \operatorname{int}\left(q_{j}\right)=\emptyset$ for $i<j$ and $q_{i} \subset[-L, L]^{2}$ are equivalent to inequalities for the $3 n$-tuple $\left(a_{i}, b_{i}, \theta_{i}\right)_{1 \leq i \leq n}$ of the shape $f_{\kappa}\left(\left(a_{i}, b_{i}, \theta_{i}\right)_{1 \leq i \leq n}\right) \leq 0$ where the functions $f_{\kappa}$, with $1 \leq \kappa \leq 2 n^{2}+2 n$, which are easily written down, are continuous but not $C^{1}$. Hence, the manifold $\mathbf{Q}_{n, c}$ is compact.

\section{Percussion and $\psi(\mathcal{C})$}

We now define the maximum inflation of a configuration of two squares $\left(q^{\prime}, q^{\prime \prime}\right) \in \mathbf{Q}_{c}^{2}$ with distinct centers: we look at the numbers $k>0$ for which $h_{k / c}\left(q^{\prime}\right) \cap h_{k / c}\left(q^{\prime \prime}\right)$ is empty. This defines an interval of $\mathbb{R}_{+}^{*}$, the supremum of which will be denoted by $\psi\left(q^{\prime}, q^{\prime \prime}\right)$. This real number is independent of $c$ and we can denote $\psi\left(q_{a^{\prime}, b^{\prime}, \theta^{\prime}, c^{\prime}}, q_{a^{\prime \prime}, b^{\prime \prime}, \theta^{\prime \prime}, c^{\prime \prime}}\right)$ by $\psi\left(a^{\prime}, b^{\prime}, \theta^{\prime}, a^{\prime \prime}, b^{\prime \prime}, \theta^{\prime \prime}\right)$. The following lemma gives the some properties of the function $\psi$ :

Lemma 1. Given two squares $q^{\prime}, q^{\prime \prime}$, with distinct centers, and diagonal $2 c$, we have the following:

(a) $\psi\left(q^{\prime}, q^{\prime \prime}\right)=\psi\left(q^{\prime \prime}, q^{\prime}\right)$.

(b) $q^{\prime} \cap q^{\prime \prime}=\emptyset$ is equivalent to $\psi\left(q^{\prime}, q^{\prime \prime}\right)>c$.

(c) For any $g \in \mathfrak{D}$, we have $\psi\left(g \cdot q^{\prime}, g \cdot q^{\prime \prime}\right)=\psi\left(q^{\prime}, q^{\prime \prime}\right)$.

(d) For any $k \in \mathbb{R}$, we have $\psi\left(h_{k / c}\left(q^{\prime}\right), h_{k / c}\left(q^{\prime \prime}\right)\right)=\psi\left(q^{\prime}, q^{\prime \prime}\right)$.

Now, it is clear that $h_{\psi\left(q^{\prime}, q^{\prime \prime}\right) / c}\left(q^{\prime}\right) \cap h_{\psi\left(q^{\prime}, q^{\prime \prime}\right) / c}\left(q^{\prime \prime}\right)$ contains at least one vertex of the two squares $h_{\psi\left(q^{\prime}, q^{\prime \prime}\right) / c}\left(q^{\prime}\right)$ and $h_{\psi\left(q^{\prime}, q^{\prime \prime}\right) / c}\left(q^{\prime \prime}\right)$; if, say, this intersection contains a vertex of $q^{\prime}$, we say that the square $q^{\prime}$ strikes the square $q^{\prime \prime}$ by inflation at $k=\psi\left(q^{\prime}, q^{\prime \prime}\right)$. We state for further reference the basic properties of this process: 


\section{Lemma 2.}

(a) Given two congruent squares with distinct centers, one of them strikes the other.

(b) The relation " $q$ ' strikes the square $q$ " by inflation at $k$ " is not symmetric in $q$ ' and $q^{\prime \prime}$.

(c) If $q^{\prime}$ strikes $q^{\prime \prime}$ and $q^{\prime \prime}$ strikes $q^{\prime}$, then $h_{\psi\left(q^{\prime}, q^{\prime \prime}\right) / c}\left(q^{\prime}\right) \cap h_{\psi\left(q^{\prime}, q^{\prime \prime}\right) / c}\left(q^{\prime \prime}\right)$ contains a common isolated vertex or the seqment joining a vertex of $q^{\prime}$ to a vertex of $q^{\prime \prime}$.

(d) If $q^{\prime}$ strikes $q^{\prime \prime}$ at $k$ and if $g \in \mathfrak{D}$, then $g \cdot q^{\prime}$ strikes $g \cdot q^{\prime \prime}$ at $k$.

(e) If $q^{\prime}$ strikes $q^{\prime \prime}$ at $k$ and if $\lambda \in \mathbb{R}$, then $h_{\lambda} \cdot q^{\prime}$ strikes $h_{\lambda} \cdot q^{\prime \prime}$ at $k$.

Lemma 3. Let $c$, s be two numbers such that $c^{2}+s^{2}=1$ and $c s \neq 0$. Among the four numbers $1-c-s, 1-c+s, 1+c+s, 1+c-s$, two are greater than one, three are positive and one is negative, the absolute value of the negative number is less than the biggest positive number among the three.

Proof. Two of the four numbers $1-(c+s), 1+(c+s), 1-(c-s), 1+(c-s)$ are greater than one. If the two others are negative we would have to say $1<\varepsilon^{\prime}(c+s)$ and $1<\varepsilon^{\prime \prime}(c-s)$ with $\varepsilon^{\prime 2}=\varepsilon^{\prime \prime 2}=1$. Let us set $c=\cos x, s=\sin x$; the product gives $1<\varepsilon^{\prime} \varepsilon^{\prime \prime}(c+s)(c-s)=\varepsilon^{\prime} \varepsilon^{\prime \prime} \cos 2 x$ which is impossible. The remainder of the proof is straightforward.

Proposition 1. The function $\psi\left(a^{\prime}, b^{\prime}, \theta^{\prime}, a^{\prime \prime}, b^{\prime \prime}, \theta^{\prime \prime}\right)$ is the maximum of $\left\{\psi_{1}, \psi_{2}\right\}$ where

$$
\begin{aligned}
& \psi_{1}=\psi_{0}\left(\begin{array}{c}
\left(a^{\prime \prime}-a^{\prime}\right) \cos \theta^{\prime}+\left(b^{\prime \prime}-b^{\prime}\right) \sin \theta^{\prime} \\
-\left(a^{\prime \prime}-a^{\prime}\right) \sin \theta^{\prime}+\left(b^{\prime \prime}-b^{\prime}\right) \cos \theta^{\prime} \\
\theta^{\prime \prime}-\theta^{\prime}
\end{array}\right), \\
& \psi_{2}=\psi_{0}\left(\begin{array}{c}
\left(a^{\prime}-a^{\prime \prime}\right) \cos \theta^{\prime \prime}+\left(b^{\prime}-b^{\prime \prime}\right) \sin \theta^{\prime \prime} \\
-\left(a^{\prime}-a^{\prime \prime}\right) \sin \theta^{\prime \prime}+\left(b^{\prime}-b^{\prime \prime}\right) \cos \theta^{\prime \prime} \\
\theta^{\prime}-\theta^{\prime \prime}
\end{array}\right),
\end{aligned}
$$

and where the function $\psi_{0}$ is defined by

$$
\begin{aligned}
\psi_{0}(a, b, \theta) & =\min _{i=1, \ldots, 4}\left\{\frac{|a|+|b|}{\left|1-\left(\operatorname{sgn}(a) \cos \theta_{i}+\operatorname{sgn}(b) \sin \theta_{i}\right)\right|}\right\} \\
& =\min _{i=1, \ldots, 4}\left\{\frac{|a|+|b|}{|1-\sqrt{2} \operatorname{sgn}(a b) \sin (\theta+\pi / 4+i(\pi / 2))|}\right\} .
\end{aligned}
$$

Proof. (i) Let $q^{\prime}, q^{\prime \prime}$ be two squares with distinct centers.

If $q^{\prime}$ strikes by inflation $q^{\prime \prime}$, then $g_{1}=\left(-a^{\prime} \cos \theta^{\prime}-b^{\prime} \sin \theta^{\prime}, a^{\prime} \sin \theta^{\prime}-b^{\prime} \cos \theta^{\prime},-\theta^{\prime}\right)$ is such that $g_{1} \cdot q^{\prime}=q_{0,0,0, c}$ and $g_{1} \cdot q^{\prime \prime}=q_{a, b, \theta}$ (say) strikes by inflation $q_{0,0,0, c}$.

Else if $q^{\prime \prime}$ strikes by inflation $q^{\prime}$, then $g_{2}=\left(-a^{\prime \prime} \cos \theta^{\prime \prime}-b^{\prime \prime} \sin \theta^{\prime \prime}, a^{\prime \prime} \sin \theta^{\prime \prime}-b^{\prime \prime} \cos \theta^{\prime \prime}\right.$, $-\theta^{\prime \prime}$ ) is such that $g_{2} \cdot q^{\prime \prime}=q_{0,0,0, c}$ and $g_{2} \cdot q^{\prime}=q_{a, b, \theta}$ (say) strikes by inflation $q_{0,0,0, c}$.

(ii) We begin with the case $\theta=0$, that is $\theta^{\prime}=\theta^{\prime \prime}$, or $q^{\prime}, q^{\prime \prime}$ parallel. Here direct inspection of the percussion process shows that it occurs at $\psi(0,0,0, a, b, 0)=\frac{1}{2}(|a|+|b|)$. 
However, the numbers given in our statement are equal to $\psi_{1}=\psi_{0}(a, b, 0)$ and $\psi_{2}=$ $\psi_{0}(-a,-b, 0)$ and, in turn, they are equal to $(|a|+|b|) \min \left\{\frac{1}{2}, \infty\right\}=(|a|+|b|) / 2$. This shows that the formula $\psi=\max \left(\psi_{1}, \psi_{2}\right)$ holds in this particular case.

(iii) We suppose now that $q_{a, b, \theta, c}$ strikes $q_{0,0,0, c}$ by inflation at $k$. Then at least one vertex of the square $h_{k / c}\left(q_{a, b, \theta, c}\right)$ belongs to the segment $[(\operatorname{sgn}(a) k, 0),(0, \operatorname{sgn}(b) k)]$ of the boundary of $h_{k / c}\left(q_{0,0,0, c}\right)$. Let this vertex be $\left(a+k \cos \theta_{i}, b+k \sin \theta_{i}\right)$, where $\theta_{i}$ is one of the four angles $\theta+i(\pi / 2)$. There exists some real number $\lambda \in[0,1]$ such that

$$
\left(a+k \cos \theta_{i}, b+k \sin \theta_{i}\right)=(\operatorname{sgn}(a)(1-\lambda) k, \operatorname{sgn}(b) \lambda k) .
$$

This system has the solution

$$
k=(|a|+|b|) \frac{1}{1-\left(\operatorname{sgn}(a) \cos \left(\theta_{i}\right)+\operatorname{sgn}(b) \sin \left(\theta_{i}\right)\right)}
$$

and

$$
\lambda=\frac{|b|+\operatorname{sgn}(b)|a| \sin \theta_{i}-\operatorname{sgn}(a)|b| \cos \theta_{i}}{|a|+|b|} .
$$

Now we observe that the least positive value of $k$ among the four preceding values obtained for $i \in\{1, \ldots, 4\}$, say $i=i_{0}$, gives rise truly to a percussion, namely membership of the $i_{0}$ th vertex of $h_{k / c}\left(q_{a, b, \theta, c}\right)$ to $h_{k / c}\left(q_{0,0,0, c}\right)$. Indeed, the other positive values of $k$ corresponding to $i_{1} \neq i_{0}$ give $h_{k / c}\left(q_{a, b, \theta, c}\right) \cap h_{k / c}\left(q_{0,0,0, c}\right) \neq \emptyset$. See the left side of Fig. 1. Hence, the value

$$
\psi_{0}(a, b, \theta)=(|a|+|b|) \min _{i=1, \ldots, 4}\left\{\frac{1}{1-\left(\operatorname{sgn}(a) \cos \left(\theta_{i}\right)+\operatorname{sgn}(b) \sin \left(\theta_{i}\right)\right)}\right\}
$$

is the least positive number such that $h_{\psi_{0}(a, b, \theta) / c}\left(q_{a, b, \theta, c}\right) \cap h_{\psi_{0}(a, b, \theta) / c}\left(q_{0,0,0, c}\right) \neq \emptyset$. Hence, when $q_{a, b, \theta, c}$ strikes $q_{0,0,0, c}$ by inflation at $k$, we have $\psi_{0}(a, b, \theta)=\psi(a, b, \theta, 0$, $0,0)$.

(iv) We note, from the previous step, that even when $q_{a, b, \theta, c}$ does not strike $q_{0,0,0, c}$ by inflation at $\psi_{0}(a, b, \theta)$, the number $\psi_{0}(a, b, \theta)$ remains well defined and is strictly less than $\psi(a, b, \theta, 0,0,0)$. Indeed, the hypothesis is merely to be said as: the system (S) has a solution, for which we get $\lambda \notin[0,1]$; hence the vertex of $h_{k / c}\left(q_{a, b, \theta, c}\right)$ that is described above lies on the straight line joining two vertices of $h_{k / c}\left(q_{0,0,0, c}\right)$. Thus, the homothetics $h_{k}\left(q_{a, b, \theta, c}\right)$ and $h_{k}\left(q_{0,0,0, c}\right)$ are disjoint, so that $\psi_{0}(a, b, \theta)<\psi(a, b, \theta, 0,0,0)$. In that
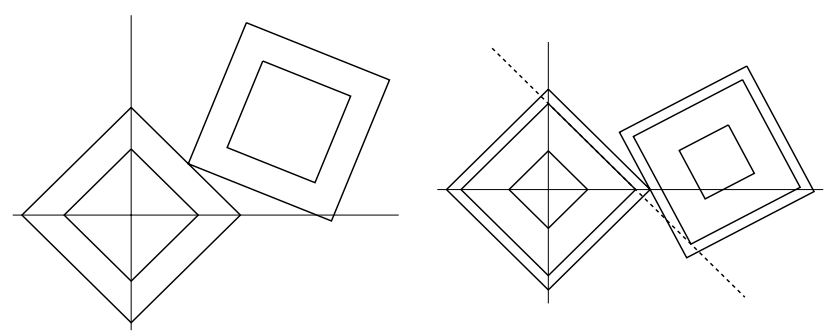

Fig. 1. The percussion process. 
case the percussion occurs "later," at

$$
\psi_{1}=\psi_{0}(-a \cos \theta-b \sin \theta, a \sin \theta-b \cos \theta,-\theta)>\psi_{2}=\psi_{0}(a, b, \theta),
$$

see the right side of Fig. 1.

(v) We consider the displacement $g_{1}$ and $g_{2}$ already defined in (i). We obtain the two numbers $\psi_{0}\left(g_{1} \cdot q_{a^{\prime \prime}, b^{\prime \prime}, \theta^{\prime \prime}}\right)$ and $\psi_{0}\left(g_{2} \cdot q_{a^{\prime}, b^{\prime}, \theta^{\prime}}\right)$. Since one of the two squares strikes the other, and by Lemma $1(\mathrm{c})$, we have $\psi\left(a^{\prime}, b^{\prime}, \theta^{\prime}, a^{\prime \prime}, b^{\prime \prime}, \theta^{\prime \prime}\right)=\max \left\{\psi_{1}, \psi_{2}\right\}$.

Remark 1. The function $\psi_{0}$ can be extended by continuity on $(0,0) \times[0, \pi / 2]$ if we set $\psi_{0}(0,0, \theta)=0$ and, correspondingly, the function $\psi\left(a^{\prime}, b^{\prime}, \theta^{\prime}, a^{\prime \prime}, b^{\prime \prime}, \theta^{\prime \prime}\right)$ can be extended to the case where $\left(a^{\prime}, b^{\prime}\right)=\left(a^{\prime \prime}, b^{\prime \prime}\right)$ by setting $\psi\left(a, b, \theta^{\prime}, a, b, \theta^{\prime \prime}\right)=0$.

Remark 2. From Lemma 3, it follows that the function $\psi_{0}(a, b, \theta)$ is well defined since the set in Proposition 1 contains three numbers, except when $\theta=0 \bmod \pi$ in which case this set is equal to $\left\{\frac{1}{2}, \infty\right\}$. Now the eight values which appear in the two underlying sets in $\psi_{1}, \psi_{2}$ contain the value $\infty$ at most twice, six positive values, and moreover can be all distinct.

Remark 3. We note an analogy between the formula of Proposition 1 and the definition of the Hausdorff distance between compact sets $A, B \subset \mathbb{R}^{d}$ :

$$
h(A, B)=\max \{g(A, B), g(B, A)\} \quad \text { where } \quad g(A, B)=\sup _{a \in A} \inf _{b \in B} d(a, b) .
$$

The function $\psi\left(q^{\prime}, q^{\prime \prime}\right)$, which is positive, symmetric and satisfies $\psi(q, q)=0$, is not however a semi-distance; the triangle inequality fails as shown in the following example. If $q_{1}=(0,0, \pi / 4), q_{2}=(1,0,0)$ and $q_{3}=(10,0,0)$, then we find

$$
\psi\left(q_{1}, q_{2}\right)+\psi\left(q_{2}, q_{3}\right)<5.08<5.85<\psi\left(q_{1}, q_{3}\right) .
$$

Corollary. The function $\psi: \mathbf{Q}_{c}^{2} \rightarrow \mathbb{R}_{+}$is continuous, and the subset of $\mathbf{Q}_{c}^{2}$, the elements of which are the couples $\left(q^{\prime}, q^{\prime \prime}\right)$ with $q^{\prime} \cap q^{\prime \prime}=\emptyset$, is open.

We define the continuous function $\psi: \mathbf{Q}_{n, c} \rightarrow \mathbb{R}_{+}$by

$$
\psi(\mathcal{C})=\min _{1 \leq i<j \leq n} \psi\left(a_{i}, b_{i}, \theta_{i}, a_{j}, b_{j}, \theta_{j}\right) .
$$

\section{Confinement and $\varphi(\mathcal{C})$}

Given a square $q_{a, b, \theta, c} \in \mathbf{Q}_{1, c}$, we are looking for the biggest homothetic square $q_{a, b, \theta, k}$ contained in $[-L, L]^{2}$. We might look at the numbers $k>0$ for which $h_{k / c}(q) \subset$ $[-L, L]^{2}$; this condition defines an interval of $\mathbb{R}_{+}^{*}$, the supremum of which will be denoted by $\varphi(a, b, \theta)=\sup \left\{k, q_{a, b, \theta, k} \subset[-L, L]^{2}\right\}$.

Proposition 2. The function $\varphi(a, b, \theta)$ is continuous on $\mathbf{Q}_{1, c}$ and defined by

$$
\varphi(a, b, \theta)=\frac{\min \{L-a, L-b, L+a, L+b\}}{\max \{|\cos (\theta)|,|\cos (\theta+\pi / 2)|\}}=\frac{L-\max \{|a|,|b|\}}{\max \{|\cos \theta|,|\sin \theta|\}} .
$$


Proof. Let $(a, b) \in[-L, L]^{2}$ and recall that $\theta_{i}=\theta+i(\pi / 2)$. The number $\varphi(a, b, \theta)$ is well defined and is the biggest value of $k$ such that $-L \leq a+k \cos \left(\theta_{i}\right) \leq L$ and $-L \leq b+k \sin \left(\theta_{i}\right) \leq L$ for all $i$; these inequalities show that $\varphi(a, b, \theta)$ is equal to

$$
\min \left\{\min _{\cos \left(\theta_{i}\right)>0} \frac{L-a}{\cos \left(\theta_{i}\right)}, \min _{\sin \left(\theta_{i}\right)>0} \frac{L-b}{\sin \left(\theta_{i}\right)}, \min _{\cos \left(\theta_{i}\right)<0} \frac{L+a}{-\cos \left(\theta_{i}\right)}, \min _{\sin \left(\theta_{i}\right)<0} \frac{L+b}{-\sin \left(\theta_{i}\right)}\right\} .
$$

We observe that for any $\theta$ we have

$$
\begin{aligned}
\min _{\cos \left(\theta_{i}\right)>0} \frac{1}{\cos \left(\theta_{i}\right)} & =\min _{\sin \left(\theta_{i}\right)>0} \frac{1}{\sin \left(\theta_{i}\right)}=\min _{\cos \left(\theta_{i}\right)<0} \frac{1}{-\cos \left(\theta_{i}\right)} \\
& =\min _{\sin \left(\theta_{i}\right)<0} \frac{1}{-\sin \left(\theta_{i}\right)}=\frac{1}{\max \{|\cos (\theta)|,|\cos (\theta+\pi / 2)|\}} .
\end{aligned}
$$

This allows us to simplify the previous values of $\varphi(a, b, \theta)$ and to obtain Proposition 2.

Note that $\varphi(a, b, \theta)$ is defined on the boundary by $\varphi(a, b, \theta)=0$ if $a= \pm L$ or $b= \pm L$. Finally, we define the continuous function $\varphi: \mathbf{Q}_{n, c} \rightarrow \mathbb{R}_{+}$by

$$
\varphi(\mathcal{C})=\min _{1 \leq i \leq n} \varphi\left(a_{i}, b_{i}, \theta_{i}\right)=\min _{1 \leq i \leq n} \frac{\min \left\{L-a_{i}, L-b_{i}, L+a_{i}, L+b_{i}\right\}}{\max \left\{\left|\cos \theta_{i}\right|,\left|\sin \theta_{i}\right|\right\}} .
$$

\section{Maximal Inflation $\omega(\mathcal{C})$ and the Graph of $\mathcal{C}$}

Now, we relate numbers $s_{n}$ with the preceding functions. Summed up, the previous results show that:

Proposition 3. The function $\omega: \mathbf{Q}_{n, c} \rightarrow \mathbb{R}_{+}$defined by

$$
\omega(\mathcal{C})=\min \{\psi(\mathcal{C}), \varphi(\mathcal{C})\}
$$

is well defined and continuous. Moreover, for every $\mathcal{C} \in \mathbf{Q}_{n, c}$, the homothetic $h_{c^{\prime} / c}(\mathcal{C})$, where $c^{\prime}=\omega(\mathcal{C})$, is an admissible configuration. If $\mathcal{C} \in \mathbf{Q}_{n, c}$ is a global maximizer for $\omega$, then we have $\omega(\mathcal{C})=\sqrt{2} L / s_{n}$.

Now, to any admissible configuration $\mathcal{C}=\left(q_{i}\right) \in \mathbf{Q}_{n, c}$ of $n$ congruent squares $q_{i}=$ $\left(a_{i}, b_{i}, \theta_{i}\right)$, we can associate a geometric graph $\Gamma(\mathcal{C})=(X, U)$. Let $\mathcal{C}$ be a configuration of $n$ squares $q_{i}$ centered at $q_{i 0}$ and with vertices $q_{i k}=q_{i 0}+c\left(\cos \theta_{i k}, \sin \theta_{i k}\right)$ with $\theta_{i k}=\theta_{i}+k(\pi / 2)$ for $k=1, \ldots, 4$. The graph $\Gamma(\mathcal{C})=(V, E)$ is non-oriented and bipartite: the set $V$ is the union of

$$
V_{c}=\left\{q_{l 0}\right\} \quad \text { and } \quad V_{s}=\left\{q_{l m}\right\}_{m \neq 0} \cap\left(\partial[-L, L]^{2} \cup \bigcup_{i<j}\left(\partial q_{i} \cap \partial q_{j}\right)\right) .
$$

In other words, the vertices in $V_{c}$ are the centers of the squares $q_{i}$, those in $V_{s}$ are the points $q_{i k}$ belonging either to $\partial q_{i} \cap \partial q_{j}$ for some $j \neq i$ or to $\partial[-L, L]^{2}$. Now, for any indices $i<j$, if the set $\partial q_{i} \cap \partial q_{j}$ is not empty, then it contains one (or two) vertex $v$ of some square, we add $v$ to $V_{s}$ and add also two (or four) edges $q_{i 0} v$ and $v q_{j 0}$ in $E$. 

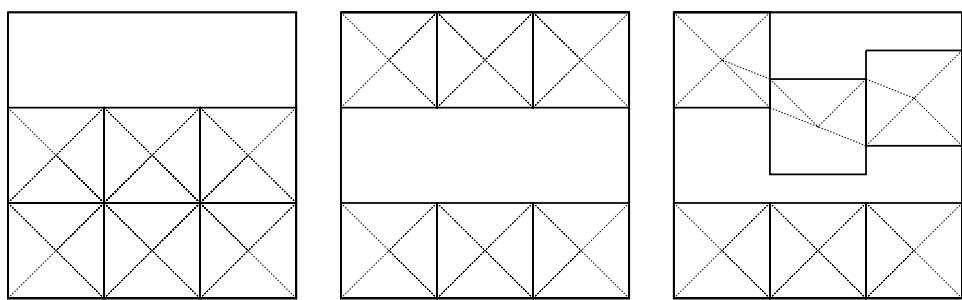

Fig. 2. Homotopic optimal packings of six squares and their graphs.

Remark 4. Whenever $E \neq \emptyset$, at least half of all the edges in $E$ have length equal to $\omega(\mathcal{C})$, while the remaining ones have length less than $\omega(\mathcal{C})$.

The following easy proposition links some of the previous definitions:

Proposition 4. Let $\mathcal{C}=\left(q_{i}\right) \in \mathbf{Q}_{n, c}$ and let $i$ be a fixed integer. We consider the following three properties:

(i) There is no edge in the graph $\Gamma(\mathcal{C})$ which starts at $q_{i 0}$.

(ii) $\psi(\mathcal{C})<\inf _{1 \leq j \leq n, j \neq i}\left\{\psi\left(q_{i}, q_{j}\right)\right\}$.

(iii) There exists an homotopy $H_{t}:[-L, L]^{2} \rightarrow[-L, L]^{2}$ such that $H_{t}\left(q_{j}\right)=q_{j}$ for all $j \neq i$ and $t \in[0,1]$, and $H_{1}\left(q_{i}\right) \neq q_{i}$, and such that $H_{t}(\mathcal{C})$ is admissible for all $t \in[0,1]$.

We have (i) $\Leftrightarrow$ (ii) and (ii) $\Rightarrow$ (iii).

The implication (iii) $\Rightarrow$ (ii) is false. For instance, for each square $q_{i}$ of the optimal packing of six congruent squares displayed in Fig. 2, (ii) is false. However, (iii) is true for the three upper squares.

When properties (i) and (ii) hold, we say that $q_{i}$ is isolated in $\mathcal{C}$.

If the graph $\Gamma(\mathcal{C})$ is connected, then for all $k, q_{k}$ is not isolated. However, the converse does not hold as is shown on the right of Fig. 2. We note finally that throughout an homotopy of configurations, neither the number of vertices nor the number of edges of the graph $\Gamma(\mathcal{C})$ remain constant.

If the graph does not encode homotopic information, it is however still well-suited to visualize contacts between squares.

\section{An Algorithm to Produce Dense Configurations}

We denote by $\mathcal{U}_{K}$ the uniform law of probability in the compact subset $K$ of $\mathbb{R}^{2}$.

The code we have used to produce dense configurations has been implemented in the language $\mathrm{C}$ and executed on a computer at CPU $800 \mathrm{MHz}$. It relies on four main procedures that we now describe. The whole program is in fact a stochastic perturbed walk through the manifolds $\mathbf{Q}_{n, c}$, with varying $c$. 
Let $\mathcal{C}=\left(q_{1}, \ldots, q_{n}\right)$ be a starting admissible configuration of $\mathbf{Q}_{n, c}$-where $c=$ $\omega(\mathcal{C})$ - the first procedure, RandomWalking, realizes some moves of randomly choosen squares $q_{i}$; these moves are done whenever the displaced square $q_{i}$ does not strike the other squares nor the boundary of $[-1,1]^{2}$. The procedure then returns a new admissible configuration $\mathcal{C}^{\prime} \in \mathbf{Q}_{n, c^{\prime}}$ with $c^{\prime} \geq c$. The larger the number $N_{a}$, the greater the chance of separating the squares.

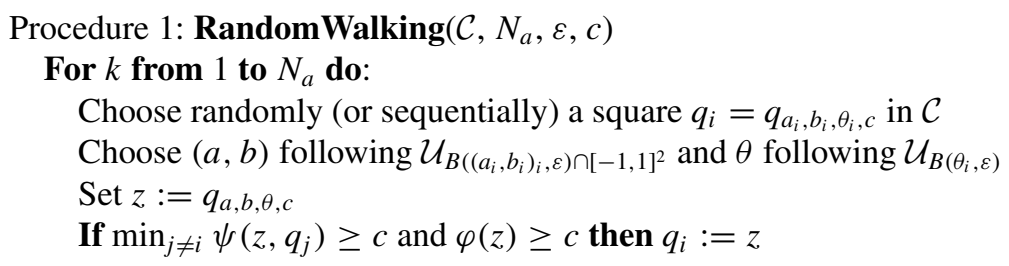

Next procedure BilliardOfSquares iterates the previous procedure; the amplitude $\varepsilon$ of the random moves is adapted through the run: at the beginning the squares are small because one of them is close to the boundary or because two squares are close to each other; then it is still possible that the squares realize big leaps. After a few steps of the loop, procedure RandomWalking does not succeed in improving $\omega(\mathcal{C})$ and one needs to decrease the amplitude $\varepsilon$ in order to accelerate the calculations. Next, the value of $\varepsilon$ follows the success or failure of procedure RandomWalking. The real number $\varepsilon_{2}$ is the threshold of the procedure.

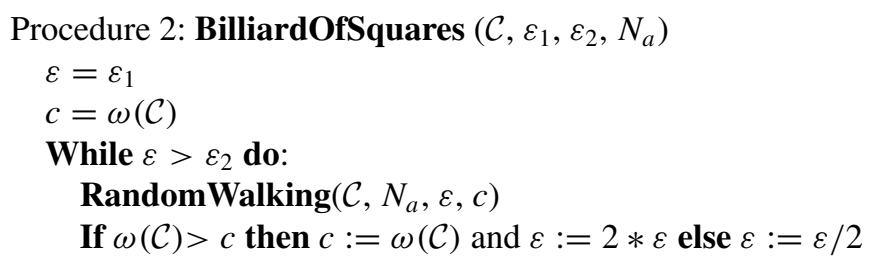

With procedure 2 we are now able to find good squares packings by sampling a large number of runs with different random starting configurations. Often, this billiard algorithm gives jammed packings with $\omega(\mathcal{C})$ close to $\sqrt{2} /([\sqrt{n}]+1)$ when the configuration obtained has just the angle $\theta=0$. When the optimal packing for a given $n$ contains more than two angles as in the case $n=17$, the chance of finding a good approximation of the configuration by procedure BilliardOfSquares becomes weak. Nevertheless, when procedure 2 gives a jammed packing topologically close to the optimal, it is possible to approach it with the third and fourth procedures. The fourth procedure, WithPerturbation, attempts to find a path toward a better configuration and this has to be done by slightly shaking the jammed configurations with procedure 3 . As procedure 2 , procedure WithPerturbations regulates the amplitude $\varepsilon$ of the moves which is also the amplitude of the perturbations. During the same process, a configuration $\mathcal{C}_{0}$ after being perturbed is improved with procedure BilliardOfSquares which gives rise to a new configuration $\mathcal{C}$. If $\omega(\mathcal{C})>\omega\left(\mathcal{C}_{0}\right)$, we keep $\mathcal{C}$ by setting $\mathcal{C}_{0}=\mathcal{C}$. In the other case, we restore the old configuration $\mathcal{C}_{0}$. 


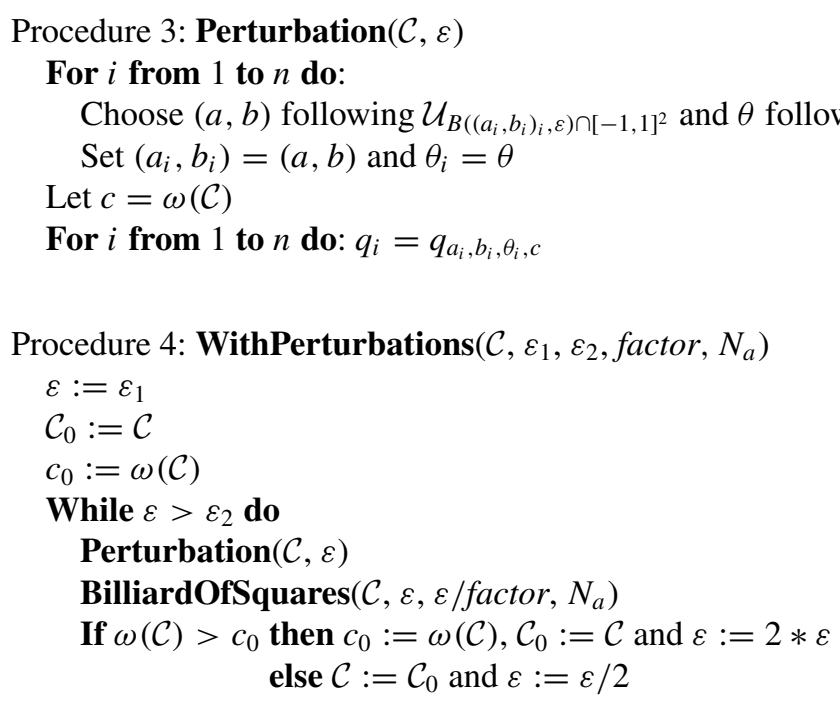

It is quite surprising that the program behaves like its disk packing version [4]. Of course, the procedures seems to be "attracted" by configurations with angle $\theta=0$ which are rarely good. Nevertheless, after running procedure BilliardOfSquares $\left(\mathcal{C}, 0.1,10^{-8}, 1000\right)$ some thousand times on random configurations, we have obtained some good starting configurations $\mathcal{C}^{*}$ for procedure WithPerturbations. The new results that we list have been obtained with the call of WithPerturbations $\left(\mathcal{C}^{*}, 0.1,10^{-12}, 1.5,1000\right)$.

\section{Some New Results}

Case $n=5$ and $n=10$. Our experiments confirm easily the optimal packing of five squares and the best known packing of ten squares, see [3] and [10] for these cases.

Case $n=11$. The best known packing is due to Trump and apparently to many other people. In [3] we find that $s_{11} \leq 3.8772$ for a packing given in Fig. 3. We have obtained this packing several times with $s_{11}=3.87708359 \ldots$, a result which is slightly better. We have also calculated that the cosine of the angle $\theta$ of the five central squares is a real root $z_{0}$ of the irreducible polynomial over $\mathbb{Q}(\sqrt{2})$ :

$$
80 z^{8}-128 \sqrt{2} z^{7}-32 z^{6}+144 \sqrt{2} z^{5}+72 z^{4}-112 \sqrt{2} z^{3}+40 z^{2}-12 \sqrt{2} z-7
$$

We have found it by eliminating with Maple a system of 14 polynomial equations, the unknowns of which are: $s=c \sqrt{2}$ is the side of the squares, $z=\cos (\theta), z^{\prime}=\sin (\theta)$, $\left(a_{i}, b_{i}\right)$ are the coordinates of the five tilted squares and $\alpha>0$ is the distance between the two upper detached squares. The distance between the two dashed sides in Fig. 3 gives the crucial relation

$$
s=\frac{4 z}{2 \sqrt{2}+5 z-z^{\prime}} .
$$




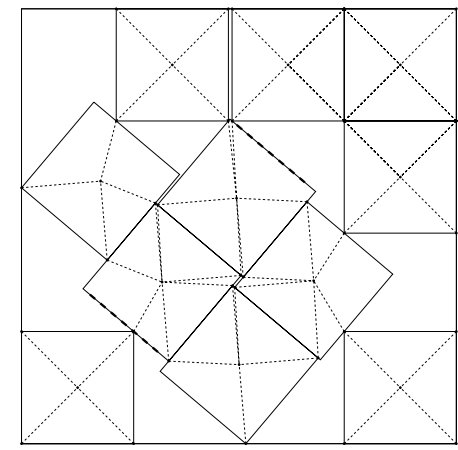

Fig. 3. $s_{11}<3.877084$.

The other equations are obtained by the requirement that some vertices $q_{i k}$ belong to sides of other squares or to the boundary of $[-L, L]^{2}$. An approximation of the root $z_{0}$ confirms the values given by our algorithm.

Case $n=17$. Friedman [3] reports on Bidwell's discovery of the packing displayed in Fig. 4 with $s_{17}<4.6755$. However, this rounded value seems to be false, since two distinct investigations of this packing lead us to the value

$$
s_{17}=4.6755300960455 \text {. }
$$

On one hand, the program described in Section 6 leads to Fig. 4 with the previous value of $s_{17}$. On the other hand, a model similar to that given in the case $n=11$ yields a polynomial system of four equations of degree 7 in $c_{1}=\cos \left(\theta_{1}\right), c_{2}=\cos \left(\theta_{2}\right)$, $s_{1}=\sin \left(\theta_{1}\right), s_{2}=\sin \left(\theta_{2}\right)$, where $\theta_{1}, \theta_{2}$ are the distinct tilted angles; solving it with 20 digits of accuracy has given the same value for $s_{17}$.

Case $n=18$. Hämäläinen and Gustafson quoted in [3] have found two different packings with $s_{18} \leq(7+\sqrt{7}) / 2$. We confirm this value with an alternative packing, displayed in

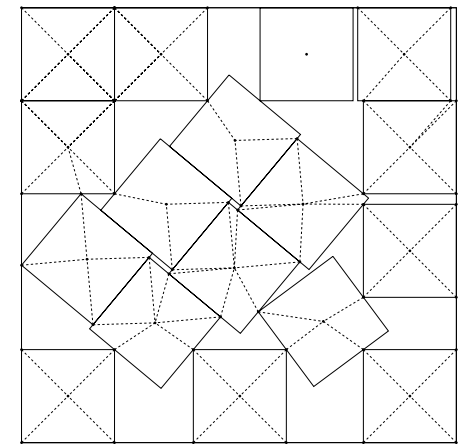

Fig. 4. $s_{17}<4.675531$. 


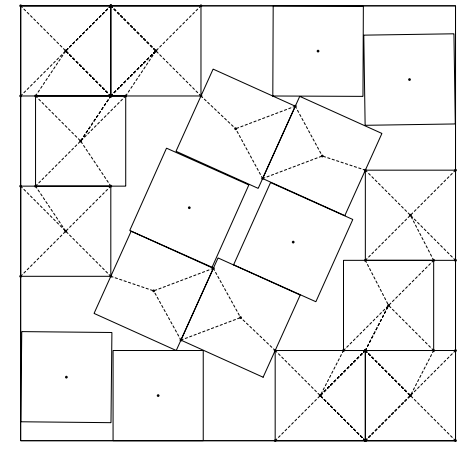

Fig. 5. $s_{18} \leq 4.822876$.

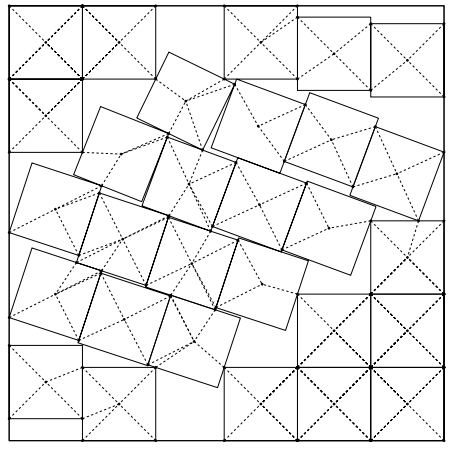

Fig. 6. $s_{29} \leq 5.934342$.

Fig. 5. It is of interest to note that six squares are isolated and six can be displaced by small translations.

Case $n=29$. We have found two packings better than Bidwell's best known packing. They realize $s_{29}<5.9648$, see Figs. 6 and 7. The upper bound becomes $s_{29} \leq$ 5.934342. Figure 6 displays a packings with squares at six different angles: $19.01^{\circ}$ (one square), $23.37^{\circ}$ (one square), $24.67^{\circ}$ (three squares), $24.74^{\circ}$ (three squares), 26.82 (seven squares) and $45^{\circ}$ (fourteen remaining). It appears to us impossible to find such a packing without a computer-aided method!

Case $n=37$. Friedman [3] has found a nice packing with just one tilted angle and $s_{37}<6.6213$. Our best packing improves this bound and we find $s_{37} \leq 6.603236$. This packing contains squares at five different angles and also six isolated squares.

Contrarily to the cases $n=11,17$ and 18, the algebraic models for the dense packings of 29 and 37 squares displayed in Figs. 6-8 are too complex to be numerically solved. However, we have verified with a sufficient accuracy that the upper bounds for $s_{29}$ and $s_{37}$ are effective. An interesting discussion of "the existence problem" for disk packings is given in [1].

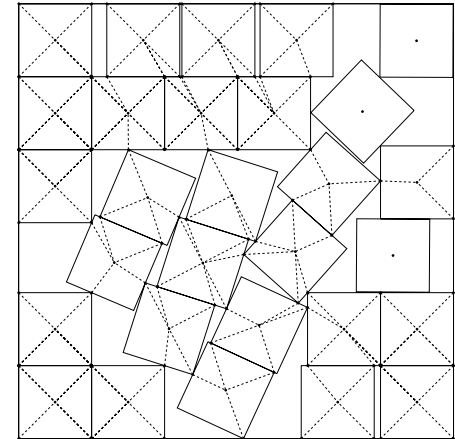

Fig. 7. $n=29, \omega(C) \leq 5.958702$.

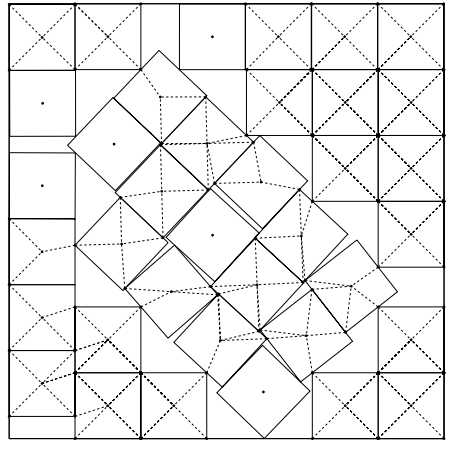

Fig. 8. $s_{37} \leq 6.603236$. 


\section{References}

1. D. Boll, J. Donovan, R. L. Graham, and B. D. Lubachevsky, Improving dense packings of equal disks in a square, Electron. J. Combin. 7 (2000), \#R46.

2. P. Erdős and R. L. Graham, On packing squares with equal squares, J. Combin. Theory Ser. A 19 (1975), 119-123.

3. E. Friedman, Packing unit squares in squares: a survey and new results, Electron. J. Combin. 7 (2000), \#DS7.

4. Th. Gensane, Dense packings of equal spheres in a cube, Report No. 188, Cahiers du L.M.P.A, June 2003.

5. R. L. Graham and B. D. Lubachevsky, Dense packings of equal disks in an equilateral triangle: from 22 to 34 and beyond, Electron. J. Combin. 2 (1995), \#A1.

6. R. L. Graham, B. D. Lubachevsky, K. J. Nurmela, and P. R. J. Östergård, Dense packings of congruent circles in a circle, Discrete Math. 181 (1998), 139-154.

7. M. Kearney and P. Shiu, Efficient packing of unit squares in a square, Electron. J. Combin. 9 (2002), \#R14.

8. B. D. Lubachevsky, How to simulate billiards and similar systems, J. Comput. Phys. 94 (1991), 255-283.

9. H. Melissen, Packing and covering with circles, Ph.D. thesis, Utrecht University, 1997.

10. W. Stromquist, Packing 10 or 11 unit squares, Electron. J. Combin. 10 (2003), \#R8.

Received April 8, 2004, and in revised form May 19, 2004. Online publication October 20, 2004. 